\title{
Methohexital Sodium
}

National Cancer Institute

\section{Source}

National Cancer Institute. Methohexital Sodium. NCI Thesaurus. Code C66117.

The sodium salt of methohexital, a rapid, short-acting barbituric acid derivative, with anesthetic activity. Methohexital binds to the chloride ionophore site of the gammaaminobutyric acid (GABA)-A/chloride ionophore receptor complex, thereby enhancing the inhibitory actions of GABA-A in the brain. This leads to synaptic inhibition, decreased neuronal excitability and induction of anesthesia. In addition, this agent decreases glutamate (Glu) responses. 